\title{
Prediction of Adsorption Free Energy Changes of Polycyclic Aromatic Hydrocarbons on Graphene Materials
}

\author{
Se Wang ${ }^{a}$, Yiwen Liu ${ }^{b}$, Ling Kang ${ }^{c}$, Yinghan Liu ${ }^{d}$, Yue Meng ${ }^{e}$ and \\ Zhuang Wang f*
}

Collaborative Innovation Center of Atmospheric Environment and Equipment Technology, Jiangsu Key Laboratory of Atmospheric Environment Monitoring and Pollution Control, School of Environmental Science and Engineering, Nanjing University of Information Science and

Technology, Nanjing 210044, China

awangse@nuist.edu.cn, byiwenliu20@163.com, 'kangling_nuist@163.com, dyinghan_liu@163.com, ${ }^{e}$ mengyue-1208@163.com, ${ }^{\mathrm{f}}$ zhuang.wang@nuist.edu.cn

*Correspondence should be addressed to Zhuang Wang: zhuang.wang@nuist.edu.cn

Keywords: Polycyclic aromatic hydrocarbons; Graphene; Adsorption free energy changes; Density functional theory

Abstract. Adsorption properties of polycyclic aromatic hydrocarbons (PAHs) on intrinsic graphene quantum dot and graphene oxide quantum dot were studied by local density approximation of density functional theory with empirical dispersion correction (LDA+DFT-D). Relevant thermochemical parameters including the changes of Gibbs free energy, enthalpy, and entropy for the complex formation were calculated. Results show that a statistically significant linear correlation $(r=0.81, p<$ $0.05)$ was found between calculated Gibbs free energy changes for the overall adsorption and experimental values. Our findings also suggest that the LDA+DFT-D method is a acceptable choice for forecasting the adsorption behavior of PAHs on graphene materials.

\section{Introduction}

Graphene materials and their derivatives (e.g. graphene oxide) which exhibit many outstanding physicochemical properties have been applied in various fields, particularly for environmental applications [1, 2]. Previous studies indicated that intrinsic graphene (G) and graphene oxide (GO) showed excellent performance in adsorption with many organic contaminates $[3,4]$. To date, there have been growing interests to develop various computational approaches to deliver useful information on molecular properties and provide an explanation on the physicochemical processes of carbon nanomaterials (CNMs) [5]. Given the huge number of existing and emerging chemical pollutants in the environment, it becomes impossible to empirically investigate the adsorption behaviors of numerous chemicals to CNMs, because of the high workload and cost [6]. It thus becomes necessary to explore a computational method that can predict the adsorption behaviors of chemicals on graphene materials.

The aim of the present study was to elucidate adsorption mechanism of PAHs by graphene materials and to develop a computational method for predicting the thermodynamic adsorption coefficient $\left(K_{\mathrm{d}}\right)$ of aqueous PAHs by the calculated Gibbs free energy changes of adsorption $\left(\Delta G_{\text {cal }}\right)$. Local density approximation (LDA) of density functional theory (DFT) with dispersion correction (LDA+DFT-D) was carried out to estimate the relevant thermochemical parameters including the changes of Gibbs free energy $(\Delta G)$, enthalpy $(\Delta H)$, and entropy $(\Delta S)$ for the complex formation.

\section{Computational Methods}

Studied materials. Eight PAHs were selected as adsorbates, including naphthalene (NAP), fluorene (FLU), phenanthrene (PHE), anthracene (ANT), pyrene (PYR), fluoranthene (FLT), benzo[a]anthracene $(\mathrm{BaA})$, and benzo[b]fluoranthene $(\mathrm{BbF})$. The constructed adsorbents included coronene, a monolayer graphene quantum dot (GQD), and a monolayer graphene oxide quantum dot (GOQD). The GQD is composed of 70 carbon atoms and 22 saturated hydrogen atoms. The GOQD 
consists of 50 carbon atoms, 11 oxygen atoms, and 24 saturated hydrogen atoms. Two smaller systems (NAP and PHE adsorbed on coronene (C), respectively) representing similar features to the studied complexes were selected to investigate the applicability of the applied method.

DFT computation. The LDA method and the LDA with the DFT-correction (DFT-D method of OBS) method were employed to calculate the isolated molecules and compounds. The exchange-correlation potential was considered for the energy calculations in the LDA with the Vosko-Wilk-Nusair functional. The conductor like screening model (COSMO) was employed to simulate the solvent (water) environment [7]. The dielectric constant for water was chosen to be 78.54. The obtained structures were optimized with the COSMO model using the LDA+DFT-D functional. Statistic thermodynamic calculation was performed by the LDA+DFT-D method at $298.15 \mathrm{~K}$ and 1 atm in water.

\section{Results and Discussion}

Adsorption parameters of two smaller systems (NAP and PHE adsorbed on C, respectively) including optimized structures, adsorption distances, adsorption energies, and entropy terms were evaluated. The distances between the adsorbate and surface obtained at the applied LDA+DFT-D and M06-2X (calculated data from Ref. [8]) levels are given in Fig. 1. The molecule-adsorbent distance obtained by the LDA+DFT-D method is slightly longer in the case of C-NAP when compared to that obtained by the M06-2X method. The molecule-adsorbent distance obtained by the LDA+DFT-D method in the case of C-PHE quite well approaches that obtained by the M06-2X method.

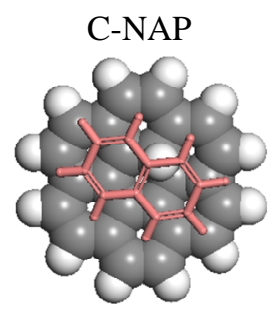

$3.11(3.34) \AA$

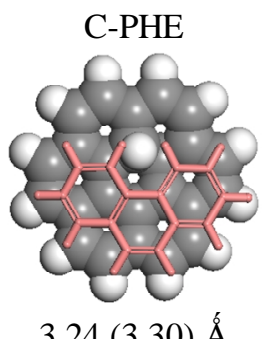

$3.24(3.30) \AA$

Fig.1. Equilibrium configurations for adsorption of naphtalene (NAP) and phenanthrene (PHE) on the coronene (C) with adsorbate-surface distances obtained by the LDA+DFT-D method in the present study and a M06-2X method (in parentheses) in ref. [8].

With regards to adsorption energy the absolute values obtained by the LDA+DFT-D method are generally greater than that obtained by the Complete basis set MP2 (MP2/CBS) and M06-2X methods (Table 1). These findings suggest that inclusion of the dispersive interaction is the most important contribution to the binding of the weakly bound systems, and does not change the shape of the interaction energy surfaces or the value of the barriers to the motion of PAH molecules on graphene materials. Furthermore, comparison also shows that the calculated adsorption enthalpies obtained by the LDA+DFT-D method correspond well to the M06-2X method (Table 1).

Table 1. Adsorption energies $\left(E_{\mathrm{a}}\right)$ and entropy terms $(T \Delta S)$ from the gas and water phase for interactions of naphtalene (NAP) and phenanthrene (PHE) with coronene

\begin{tabular}{|c|c|c|c|c|c|c|c|}
\hline \multirow{3}{*}{ PAHs } & \multirow{2}{*}{\multicolumn{3}{|c|}{$\frac{E_{\mathrm{a}}(\mathrm{kcal} / \mathrm{mol})}{\text { Gas phase }}$}} & \multicolumn{4}{|c|}{$T \Delta S(\mathrm{kcal} / \mathrm{mol})$} \\
\hline & & & & \multicolumn{2}{|c|}{ Gas phase } & \multicolumn{2}{|c|}{ Water solution } \\
\hline & $\begin{array}{l}\text { LDA+ } \\
\text { DFT-D }\end{array}$ & $\mathrm{MP}^{2} / \mathrm{CBS}^{a}$ & M06- $2 X^{a}$ & $\begin{array}{l}\text { LDA+ } \\
\text { DFT-D }\end{array}$ & M06- $2 X^{a}$ & $\begin{array}{l}\text { LDA+ } \\
\text { DFT-D }\end{array}$ & M06- $2 X^{a}$ \\
\hline NAP & -43.76 & -25.8 & -13.3 & -14.6 & -14.1 & -14.6 & -14.3 \\
\hline PHE & -47.14 & -30.5 & -15.5 & -16.0 & -13.3 & -15.7 & -13.2 \\
\hline
\end{tabular}

${ }^{a}$ Computational data in ref. [8]. 
In general, the comparison demonstrates that the LDA+DFT-D method may have similar computing power to the ultrahigh-accuracy computing methods such as the MP2 method and the M06-2X method. In addition, the DFT-D method is a relatively cheap and acceptable choice for calculating weak interactions in our systems. As mentioned in many reports, this method is now widely used to deal with adsorption systems with weak interactions $[8,9]$.

For the GQD-PAH and GOQD-PAH systems, $\Delta G_{\text {cal }}$, enthalpy ( $\left.\Delta H_{\text {cal }}\right)$, and $\Delta S$ were calculated by the following equations:

$$
\begin{gathered}
\Delta G_{\text {cal }}=G_{\text {complex }}-G_{G Q D / G O Q D}-G_{\mathrm{PAH}} \\
\Delta H_{\text {cal }}=H_{\text {complex }}-H_{G Q D / G O Q D}-H_{\mathrm{PAH}} \\
\Delta S=\left(\Delta H_{\text {cal }}-\Delta G_{\text {cal }}\right) / \mathrm{T}
\end{gathered}
$$

where $H$ is the sum of electronic and thermal enthalpies and GQD is the sum of electronic and thermal free energies for the optimized configurations.

The simulated $\Delta G_{\text {cal }}, \Delta H_{\text {cal }}$, and $\Delta S$ values in water are listed in Table 2. For the GQD-PAH and GOQD-PAH systems, all the values of $\Delta H_{\text {cal }}$ and $\Delta S<0$, implying that the adsorption is exothermic

\begin{tabular}{|c|c|c|c|c|c|c|c|c|}
\hline \multirow[b]{2}{*}{ PAHs } & \multicolumn{4}{|c|}{ GQD-PAHs } & \multicolumn{4}{|c|}{ GOQD-PAHs } \\
\hline & $\begin{array}{c}\Delta H_{\mathrm{cal}}{ }^{a} \\
\mathrm{kcal} / \mathrm{mol}\end{array}$ & $\begin{array}{c}\Delta S \\
\mathrm{cal} /(\mathrm{mol} \cdot \mathrm{K})\end{array}$ & $\begin{array}{c}\Delta G_{\mathrm{cal}}{ }^{a} \\
\mathrm{kcal} / \mathrm{mol}\end{array}$ & $\begin{array}{c}\Delta G_{\exp }{ }^{b} \\
\mathrm{kcal} / \mathrm{mol}\end{array}$ & $\begin{array}{c}\Delta H_{\text {cal }}{ }^{a} \\
\mathrm{kcal} / \mathrm{mol}\end{array}$ & $\begin{array}{c}\Delta S \\
\mathrm{cal} /(\mathrm{mol} \cdot \mathrm{K})\end{array}$ & $\begin{array}{c}\Delta G_{\mathrm{cal}}{ }^{a} \\
\mathrm{kcal} / \mathrm{mol}\end{array}$ & $\begin{array}{c}\Delta G_{\exp }{ }^{b} \\
\mathrm{kcal} / \mathrm{mol}\end{array}$ \\
\hline NAP & -51.34 & -45.38 & -37.81 & -5.44 & -55.81 & -37.29 & -44.70 & -5.76 \\
\hline FLU & -64.95 & -46.15 & -51.19 & - & -61.86 & -39.54 & -50.07 & - \\
\hline PHE & -69.47 & -47.90 & -55.19 & -7.73 & -60.03 & -50.81 & -44.88 & -7.61 \\
\hline ANT & -67.31 & -52.96 & -51.52 & - & -60.96 & -45.30 & -47.46 & - \\
\hline PYR & -77.24 & -58.02 & -59.94 & -8.62 & -65.87 & -43.15 & -53.00 & -9.31 \\
\hline FLT & -74.27 & -49.51 & -59.51 & - & -54.24 & -49.78 & -39.40 & - \\
\hline $\mathrm{BaA}$ & -99.84 & -60.57 & -81.78 & - & -72.52 & -55.13 & -56.09 & - \\
\hline $\mathrm{BbF}$ & -94.22 & -53.66 & -78.22 & - & -63.62 & -48.22 & -49.24 & - \\
\hline
\end{tabular}
and associated with ordering. The negative $\Delta G_{\text {cal }}$ values suggest that the adsorption is spontaneous.

Table 2. Thermodynamic parameters of the PAH adsorption systems

${ }^{a}$ The $\Delta H_{\text {cal }}$ and $\Delta G_{\text {cal }}$ values were computed using the LDA+DFT-D method.

${ }^{b}$ The $\Delta G_{\text {exp }}$ values were calculated from the experimental data [4].

The $\Delta G_{\text {exp }}$ values of three PAHs (NAP, PHE, and PYR) were calculated from one batch of experimental adsorption isotherms [4]. The experimental data of fitting using the Freundlich equation was used to obtain $\Delta G_{\text {exp }}$. Due to the given Freundlich constants are not the ones given in the fitting, the real $K_{\mathrm{d}}$ values used for the calculation of $\Delta G_{\text {exp }}$ were computed according to the following equations.

$$
\begin{aligned}
& \theta=k a^{\frac{1}{n}}=\frac{q_{e}}{q_{\max }} \\
& q_{e}=q_{\max } \times K_{\mathrm{d}}^{\frac{1}{n}} \times a^{\frac{1}{n}} \\
& a=\gamma c / c^{\theta} \\
& q_{e}=K_{f} c_{e}^{N}
\end{aligned}
$$

where $\theta$ refers to the coverage ratio $[10,11] ; k$ is a parameter in the equilibrium equation and $k=K_{\mathrm{d}}^{\frac{1}{n}}$; $a$ is the activity of the adsorbate in solution at equilibrium. The equilibrium concentration of adsorbate in solution is less than $10^{-3} \mathrm{~mol} / \mathrm{L}$, and the $\gamma$ is activity coefficients adsorbates, and it approaches 1 as an approximate value; $q_{\mathrm{e}}$ is adsorption quantity and $q_{\max }$ is the maximum adsorption quantity at the 
equilibrium; $c$ is the concentration of adsorbates in solution at equilibrium and $c^{\theta}$ is the molar concentration of the standard solution; $n$ and $N$ are exponential coefficients.

To obtain real $K_{\mathrm{d}}$ value, a relationship needed to be created between the theoretical equation and the fitting equation.

$$
N=\frac{1}{n}
$$

Since the unit of ${ }^{c_{e}}$ used in the fitting is not $\mathrm{mol} / \mathrm{L}$ but $\mathrm{mg} / \mathrm{L}, \mathrm{c}$ was calculated by

$$
c=\frac{c_{e} \times 10^{-3}}{M}
$$

where $M$ is molecular weigh. The final equation for the real $K_{\mathrm{d}}$ value is

$$
K_{\mathrm{d}}=\left(\frac{K_{f}}{q_{\max }}\right)^{\frac{1}{N}} \times 1000 \times M \times c^{\theta}
$$

The $\Delta G_{\text {exp }}$ for the interaction was calculated by

$$
\Delta G_{\exp }=-R T \ln K_{\mathrm{d}}
$$

where $R$ refers to the universal gas constant $(8.314 \mathrm{~J} / \mathrm{mol} \cdot \mathrm{K})$, and $T$ refers to the experimental temperature (in the involved experiment, the room temperature is $298.15 \mathrm{~K}$, the same as we setted in the calculation).

As presented in Fig. 2, for the GQD-PAH systems, the $\Delta G_{\text {exp }}$ values correlate with the $\Delta G_{\text {cal }}$ values significantly, i.e.,

$$
\Delta G_{\text {exp }}=-0.10+0.14 \Delta G_{\text {cal }}(r=1.00, p<0.05)
$$

However, for the GOQD-PAH systems, the $\Delta G_{\exp }$ values correlate with the $\Delta G_{\text {cal }}$ values insignificantly $(r=0.86, p>0.05)$.

For the overall systems including the GQD-PAHs and GOQD-PAHs, a significant correlation can be identified between $\Delta G_{\text {exp }}$ and $\Delta G_{\text {cal }}$, i.e.,

$$
\Delta G_{\text {exp }}=-0.12+0.15 \Delta G_{\text {cal }}(r=0.81, p<0.05)
$$

As experimental data are only available for the adsorption of three PAHs by GQD and GOQD, the obtained correlation can be employed to estimate $K_{\mathrm{d}}$ values of other PAHs on graphene materials, especially for the aromatics like PAHs, by calculating the $\Delta G_{\text {cal }}$ values.

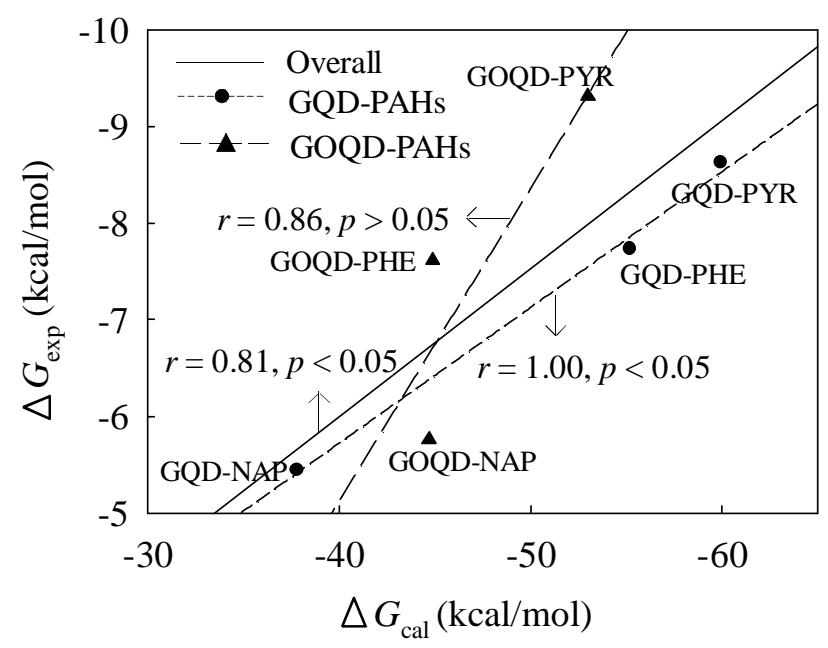

Fig. 2. Correlation between the experimental and the calculated data of $\Delta G$

It can be also seen from Fig. 2 that the absolute $\Delta G_{\text {cal }}$ values are higher than the absolute $\Delta G_{\text {exp }}$ values. This may be caused by the uncertainties of the calculation method and the implicit solvent model [6]. In addition, the pristine GQD and GOQD with an ideal homogeneous outside surface were selected in our DFT-D calculations. However, the GQD and GOQD nanosheets with surface 
functional groups or defects were employed in the experiment [4]. Hence, these factors may cause the deviation of the $\Delta G_{\text {cal }}$ values from the $\Delta G_{\text {exp }}$ values.

\section{Conclusions}

In summary, the LDA+DFT-D method is a relatively low-cost and acceptable choice for evaluating weak interactions in our systems. It was also found that there is a significant linear correlation between the calculated microscopic $\Delta G_{\text {cal }}$ and the macroscopic $\Delta G_{\text {exp }}$ values, which may be applied to forecast the adsorption behavior of other PAHs and analogues in the absence of experimental adsorption data.

\section{Acknowledgments}

This work was financially supported by the Natural Science Foundation of Jiangsu Province (BK20150891 and BK20140987), the Startup Foundation for Introducing Talent of Nanjing University of Information Science and Technology (2015r011), and the University Innovation Research/Training Program of Jiangsu Province (201610300096).

\section{References}

[1] M. S. Mauter and M. Elimelech, "Environmental applications of carbonbased nanomaterials," Environmental Science and Technology, vol. 42, no. 16, pp. 5843-5859, 2008.

[2] Q. Liu, J. B. Shi, and G. B. Jiang, "Application of graphene in analytical sample preparation," TrAC-Trends in Analytical Chemistry, vol. 37, pp. 1-11, 2012.

[3] L. Ji, W. Chen, Z. Xu, S. Zheng, and D. Zhu, "Graphene nanosheets and graphite oxide as promising adsorbents for removal of organic contaminants from aqueous solution," Journal of Environment Quality, vol. 42, no. 1, pp. 191-198, 2013.

[4] J. Wang, Z. Chen, and B. Chen, "Adsorption of polycyclic aromatic hydrocarbons by graphene and graphene oxide nanosheets," Environmental Science and Technology, vol. 48, no. 9, pp. 4817-4825, 2014.

[5] Z. Wang, J. Chen, Q. Sun, and W. J. Peijnenburg, " $\mathrm{C}_{60}-\mathrm{DOM}$ interactions and effects on $\mathrm{C}_{60}$ apparent solubility: a molecular mechanics and density functional theory study," Environment International, vol. 37, no.6, pp. 1078-1082, 2011.

[6] M. Zou, J. Zhang, J. Chen, and X. Li, "Simulating adsorption of organic pollutants on finite $(8,0)$ single-walled carbon nanotubes in water," Environmental Science and Technology, vol. 46, no. 16, pp. 8887-8894, 2012.

[7] A. Klamt, "Conductor-like screening model for real solvents: a new approach to the quantitative calculation of salvation phenomena," Journal of Physical Chemistry, vol. 99, no. 7, pp. 2224-2235, 1995.

[8] A.M. Scott, L. Gorb, E.A. Mobley, F.C. Hill, and J. Leszczynski, "Predictions of Gibbs free energies for the adsorption of polyaromatic and nitroaromatic environmental contaminants on carbonaceous materials: efficient computational approach," Langmuir, vol. 28, no. 37, pp. 13307-13317, 2012.

[9] N. Ding, X. Chen, and C.L. Wu, "Interactions between polybrominated diphenyl ethers and graphene surface: a DFT and MD investigation," Environmental Science: Nano, vol. 1, pp. 55-63, 2014.

[10] Z. Q. Zhang, Z. F. Meng, and Y. P. Zhang, "Calculation of thermodynamic parameters for Freundlich and Temkin isotherm models," Pedosphere, vol. 9, no. 4, pp. 319-322, 1999.

[11] Y. Liu, "Journal of Chemical \& Engineering Data," vol. 54, no.7, pp.1981-1985, 2009. 